\title{
Progress in Physics of the Cosmos
}

\author{
M. Zafar Iqbal \\ Department of Physics, COMSATS Institute of Information Technology, Islamabad, Pakistan \\ Email: mziqbal@comsats.edu.pk
}

Received 4 March 2015; accepted 5 May 2015; published 8 May 2015

Copyright (C) 2015 by author and Scientific Research Publishing Inc.

This work is licensed under the Creative Commons Attribution International License (CC BY).

http://creativecommons.org/licenses/by/4.0/

(c) (i) Open Access

\section{Abstract}

Study of the Cosmos, at best, is considered a semi-scientific discipline, primarily because the laboratory for carrying out measurements and tests of theories (the Cosmos) has been largely inaccessible for centuries. The cosmic vista into the yonder, however, continued to fascinate humankind due to its inherent beauty and sheer curiosity. The invention of the optical telescope more than five centuries back, however, led to the opening of observational cosmology as a scientific discipline with firm experimental basis. However, the investigations based on visible light posed obvious limitations for the range of such observational cosmology. The advent of the radio telescope in the first half of the 20th century marked a fundamental new step in the progress of this branch of science. There has been no looking back in the march of knowledge in the discipline since then. A whole new vista was laid bare as a result of this development, leading to the discovery of altogether new celestial objects, such as quasars and pulsars and still newer galaxies. The parallel progress of the physics of fundamental constituents of the material world and their interactions led to an interesting merger of these two branches of physical sciences, yielding absolutely astounding knowledge of the nature and evolution of the Universe. New concepts of dark energy and dark matter thought to constitute the dominant share of the Universe were brought to light as a result of these new observations and theoretical ideas. This brief article aims to provide an overview of these exciting developments in the field of cosmology and the associated physics.

\section{Keywords}

Radio Astronomy, Cosmic Microwave Background, Dark Energy, Dark Matter, Swirling B Modes

\section{Introduction}

The birth and evolution of the Universe, of which we are one of the tiniest parts, have been an enigma and a challenge to unravel ever since humankind started taking a rational view of things around him. For a long time, Cosmology, as a branch of Physics, (Astrophysics) has been regarded more as soft science based on guesswork 
and heuristic ideas devoid of any solid scientific base, largely due to absence of concrete observational evidence. However, as the means to explore the Universe came within man's reach as a result of developments in various branches of physics and science, in general, more and more hard evidence on the different celestial objects and the universe beyond our immediate neighborhood in the cosmos started emerging and the revelation of extraordinary nature and properties of these objects fired the imagination of physicists and scientists, in general, to reach beyond our immediate galaxy, the Milky Way. A brief introduction to how it became possible to do so and with what scientific tools would be provided in the early part of this report. As amazing new facts about the nature of the astronomical bodies and systems inhabiting the cosmos emerged as a result of availability of these advanced means to access distances hitherto thought beyond our means to explore, a coherent picture of the origin and evolution of the universe started to emerge by the late 20th century, culminating in the so called Standard Model of Cosmology. This was greatly helped by exciting new developments in the realm of particle physics heralded by the advent of large and expensive accelerator machines, allowing exploration of the structure of the tiniest of the constituents of matter and hence of our universe. Although these developments led to a lot of clarity in our understanding of the science of cosmology, some new enigmas also emerged in their wake. Some of these unanswered questions will be touched upon towards the end of this article.

\section{Historical Background}

\subsection{Early Explorations}

Our understanding of the cosmos has, in general, been impeded by the lack of experimental means to observe it. These observations were limited to the range of the optical telescope invented by Galileo in 1609, to begin with. However, this scientific development, momentous though it was, could only extend our knowledge of the cosmos (at whatever rudimentary level possible), not far beyond our own solar system or the Milky Way galaxy, in which it lies. Our observations and, therefore, directly measured data, which are the basis of any scientific understanding of a system, could, at most, cover a miniscule fraction of the universe as long as we were limited to the light (radiation) spanning only the visible part of the optical spectrum. Clearly, we had to extend our capability of observation to the use of 'light' beyond this tiny band of wavelengths, if we intended to explore the elements of the Universe well beyond, since it was established soon after the discovery of the electromagnetic waves, which carry information about their origin and whatever lies in between the observer and such sources, that the cosmos was full of electromagnetic wave signals, harking for research and investigation to establish their nature and origins, just as visible light gave us a clue of what we were looking at around us and our earth. The development of electronic technology and the sensitive tools to detect even weak signals around us first brought us to the advent of radio astronomy. This made it possible for mankind to extend his sphere of exploration of the Cosmos to the 'invisible' (to the human eye or the optical telescope) part of the universe.

\subsection{Radio Astronomy}

Radio astronomy started with a serendipitous discovery in 1932 by Jansky [1], a scientist at the Bell Telephone Laboratories, of radio waves coming from space. Development of radar and allied technology during the $2 \mathrm{nd}$ World War led to a boost to this branch of science, bringing into limelight focused studies to look for radio wave sources from around the Universe, using specially designed high resolution radio telescopes, yielding remarkable results to enrich our knowledge of the universe. The power of radio astronomy is well demonstrated by the well-resolved structure, with much enhanced clarity, in the equatorial plane of our Milky Way galaxy, as observed by a radio telescope in comparison to that observed by visible light, as shown in Figure 1. Unlike visible light, radio waves are not absorbed by clouds, interstellar dust, or the atmosphere of the Earth. They, thus, provide a superior means of exploring distant celestial objects in the universe, as compared to the ordinary visible light waves. The original radio wave signal picked up by Jansky turned out to originate from the centre of the Milky Way. Large diameter dish antennae telescopes had to be constructed to achieve relatively high resolutions for meaningful exploration of the cosmos. Whereas radio wave emission from the Sun was first observed [2] in 1942, the first distant objects, i.e., the galaxies Centaurus A and M87 and the Crab Nebula, shown in Figure 2, were identified as strong radio sources in 1949 [3].

Planetary science of our solar system benefitted from the development of the radio telescope greatly, since, although planets only reflect visible light, they may, however, emit radio waves, leading to their detailed studies 

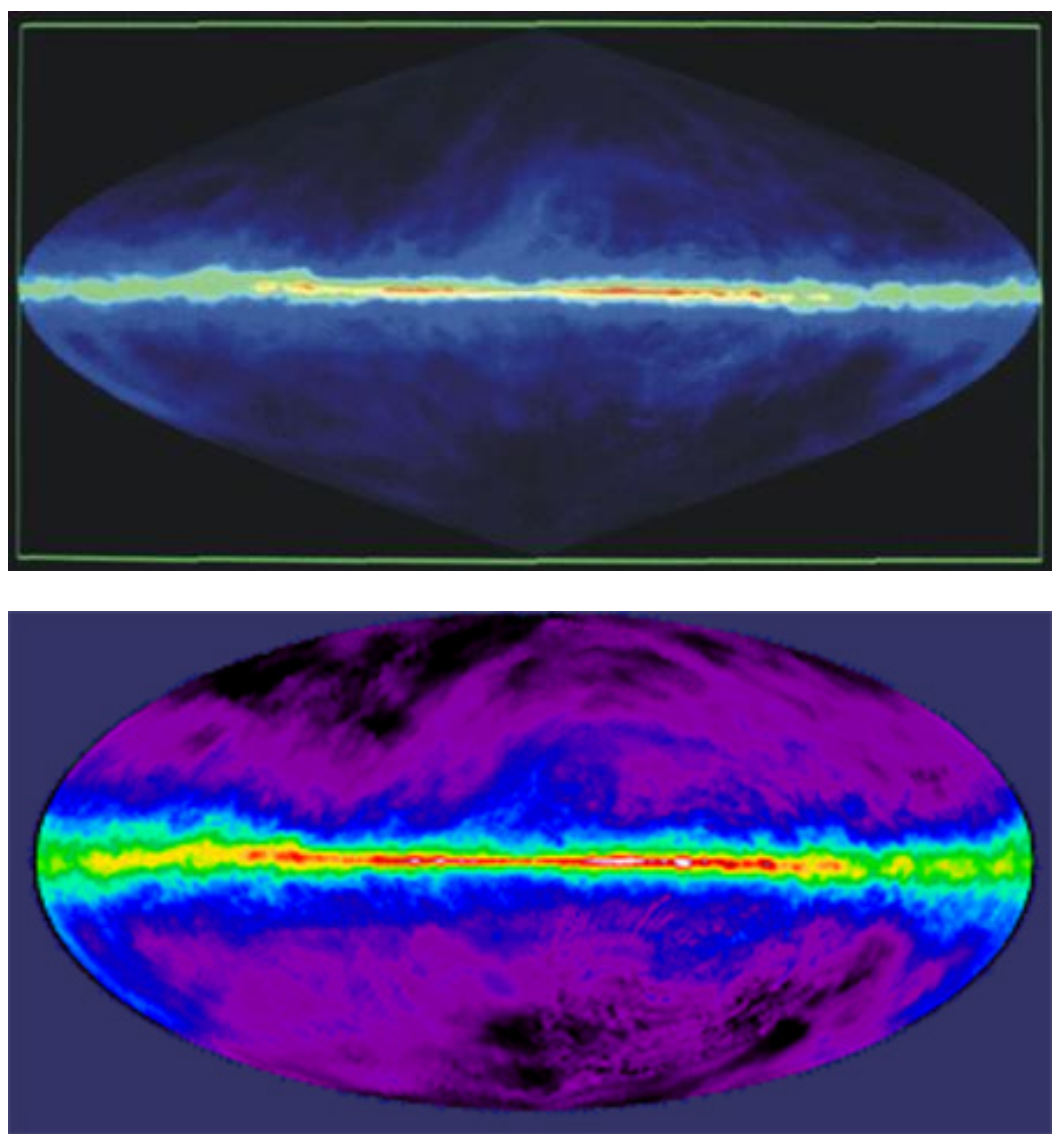

Figure 1. (Top) The Milky Way galactic plane under visible light. (Bottom) The structures of galactic plane become clear in $21 \mathrm{~cm}$ hydrogen spectral line observations. (Photo credit: NASA). Source: The National Radio Astronomy Observatory (NRAO). http://www.physics.hku.hk/ astro/hkusrt/radio astro.html

using the new type of telescopes. The surface temperature of Venus was measured from such observations [4] as were some initial explorations made on radio wave emissions, both continuous and pulsed, from Jupiter [5]. Studies based on the $21 \mathrm{~cm}$ hydrogen line emission, first detected in 1951 [6] [7] have been extremely useful for the exploration of the interstellar matter, which largely consists of neutral hydrogen gas. Such studies, for example, led to the observation of the many spiral arms of our galaxy [8]-[11] finer than those of other galaxies. The formation and evolution of stars from regions of interstellar space containing other gases, which are the sources of radio waves, have been widely studied with radio telescopes, yielding exceptionally useful information on star birth. New galaxies, such as Cygnus A, have been found to be a million times brighter than Milky Way in radio-wave region [12] [13] of emission spectrum, with large lobes of radio-wave emission (Figure 3) around the central region emitting in the optical domain - detailed studies showed that the outer radio wave lobes are only 3 million years old as compared to the central region going back to 10 billion years. Radio astronomy has revealed altogether new facts about the Cosmos, such as the existence of quasi-stellar objects (quasars) in 1960 [14]-[16], which emit brightly both in the visible and the radio frequency region of the electromagnetic wave spectrum. These strange objects were at the centre of fascinating studies by astronomers, as well as physicists, during the 1960s; detailed redshift studies indicated them to be just about the farthest objects in the universe at the time, some 12 billion light years from Earth. As such, they held the promise of yielding information on the early stages of the Universe. The discovery of pulsars [17], following in 1967, showed how rotating neutron stars, some of the most densely packed material objects in the universe, could lead to the emission of pulses of radio waves at such an amazingly regular interval (33 milliseconds for the crab nebula pulsar), that they can be used as "astronomical clocks" with the highest precision. These wonderful lighthouse-like objects have inspired a great, in-depth understanding of the Universe by providing detailed insight into the life cycle of the stars. The 


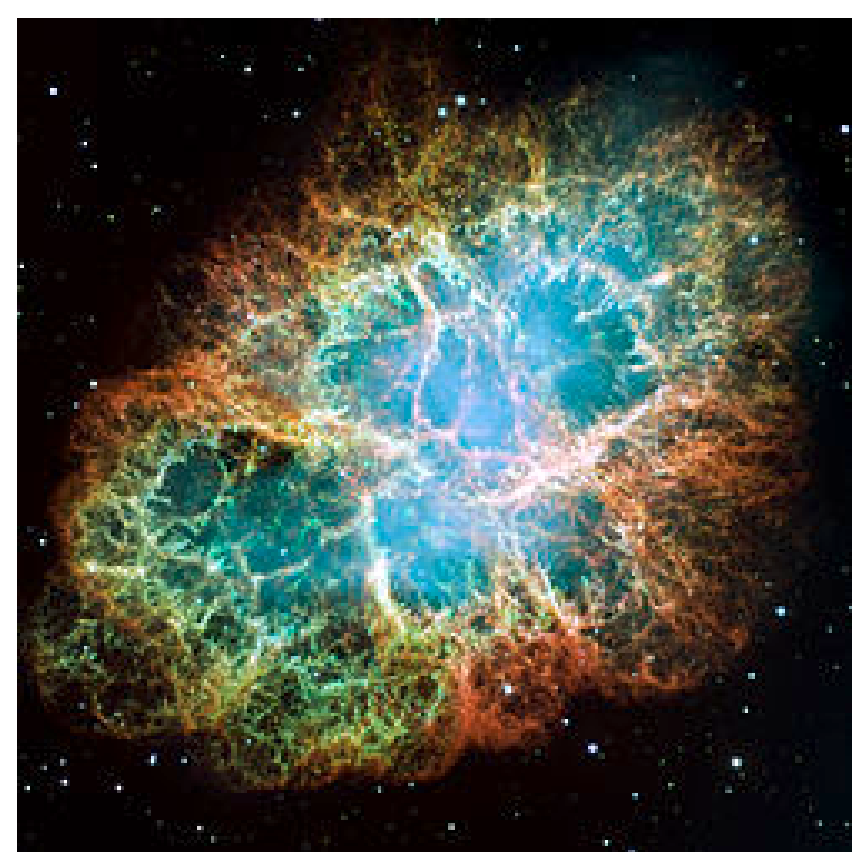

Figure 2. Hubble Space Telescope photograph of the Crab Nebula $(2005)^{1}$. http://en.wikipedia.org/wiki/Crab_Nebula

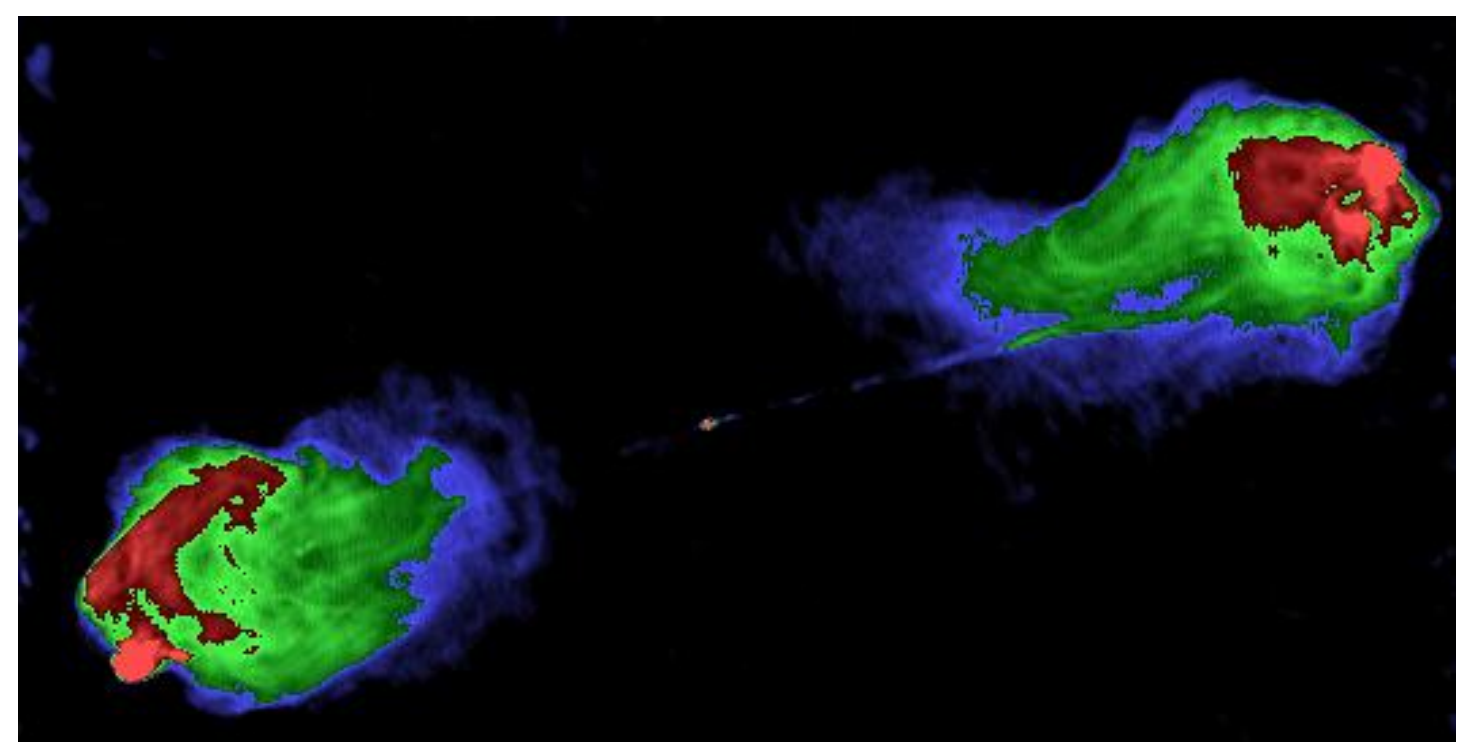

Figure 3. 5-GHz radio image of Cygnus A (3C405). http://en.wikipedia.org/wiki/Cygnus A\#cite note-3

${ }^{1}$ This is a mosaic image, one of the largest ever taken by NASA's Hubble Space Telescope of the Crab Nebula, a six-light-year-wide expanding remnant of a star's supernova explosion. Japanese and Chinese astronomers recorded this violent event nearly 1000 years ago in 1054, as did, almost certainly, Native Americans. The orange filaments are the tattered remains of the star and consist mostly of hydrogen. The rapidly spinning neutron star embedded in the center of the nebula is the dynamo powering the nebula's eerie interior bluish glow. The blue light comes from electrons whirling at nearly the speed of light around magnetic field lines from the neutron star. The neutron star, like a lighthouse, ejects twin beams of radiation that appear to pulse 30 times a second due to the neutron star's rotation. A neutron star is the crushed ultra-dense core of the exploded star. The Crab Nebula derived its name from its appearance in a drawing made by Irish astronomer Lord Rosse in 1844, using a 36-inch telescope. When viewed by Hubble, as well as by large ground-based telescopes such as the European Southern Observatory's Very Large Telescope, the Crab Nebula takes on a more detailed appearance that yields clues into the spectacular demise of a star, 6500 light-years away. The newly composed image was assembled from 24 individual Wide Field and Planetary Camera 2 exposures taken in October 1999, January 2000, and December 2000. The colors in the image indicate the different elements that were expelled during the explosion. Blue in the filaments in the outer part of the nebula represents neutral oxygen, green is singly-ionized sulfur, and red indicates doubly-ionized oxygen. 
development of radio astronomy, thus, expanded the horizon of our knowledge and understanding of the Universe from the elements of our own solar system and its planets to stars and other objects in our galaxy, to interstellar space and galaxies far beyond.

\section{Cosmic Microwave Background}

The discovery of cosmic microwave background (CMB) radiation in 1964 [18] is one of the most significant milestones in the development of the science of cosmology in the modern era, based on observations in radio astronomy. The serendipitous detection of this all pervading diffuse microwave radiation, found to have isotropic distribution over entire space, corresponding to a black body temperature of $2.73 \mathrm{Kelvin}$, although a mind boggling puzzle to start off with, was soon interpreted as the remnant of the massive explosion that accompanied the creation of the Universe, estimated to have taken place some 13.7 billion years ago. This event, heralding enormous release of energy, came to be dubbed as the big bang with time. The point in space of this event corresponded to a singularity from which radiation emanated in all directions, eventually transforming into matter (elementary particles of all descriptions constituting the material universe) as it cooled down. The material universe is believed to be expanding as per a law attributed to Hubble, but first derived from Einstein's equations of General Relativity by Georges Lemaitre in 1927, which states that all cosmological bodies are receding from each other with a velocity proportional to their distance from each other. The proportionality constant which was first measured by Hubble in 1929 has come to be known as the Hubble constant and its most recent and accurate value is $\mathrm{H}_{0}=74.3 \pm 2.1(\mathrm{~km} / \mathrm{s}) / \mathrm{Mpc}$, pc being the symbol for a parsec, an astronomical unit of distance equal to $3.09 \times$ $10^{13} \mathrm{~km}$. The crab nebula, for instance, is receding from us at a rate of $\sim 1500 \mathrm{~km} / \mathrm{s}$. The discovery of the cosmic microwave background radiation was the first definitive evidence for the big bang origin of our expanding universe.

\section{Structure of the Universe}

\section{1. $\mathrm{COBE}$}

Whereas the uniformity of $\mathrm{CMB}$ in all directions in the cosmos seemed to be a striking feature of its discovery, detailed later studies based on COBE (Cosmic Background Explorer), a satellite in the Explorer series launched by NASA on November 18, 1989 in a sun-synchronous orbit, revealed strong evidence for an all important anisotropy in CMB, first announced on April 23, 1992 [19]. The famous full map of this anisotropic distribution of CMB obtained by COBE is shown in Figure 4. These fluctuations in CMB around the sky are extremely weak (about one part in 100,000 as compared to the 2.73 Kelvin average temperature of the radiation field), explaining why the initial measurements found a virtually isotropic microwave background, regardless of the position in the Cosmos. This anisotropy, clearly, hinted at the local density fluctuations of the Universe at or close to the time of the big bang, as evidenced today by the existence of galaxies interspersed by empty space. This was the first indication of the reason for the existence of today's structure in the Universe. This important observation led to very significant advances in our understanding of the formation of stars, galaxies of stars and their clusters and, hence, the evolution of the Universe. This significance of the discovery has been recognized by the award of the Nobel Prize for Physics in 2006 to John C. Mather of the NASA Goddard Space Flight Center and George F. Smoot at the University of California, Berkeley, two of COBE's principal investigators. According to the Nobel Prize committee, "the COBE-project can also be regarded as the starting point for cosmology as a precision science" [20].

\subsection{WMAP}

The COBE mission was followed by the Wilkinson Microwave Anisotropy Probe (WMAP) launched on June 30, 2001 with the objective of obtaining a more precise full sky map of the anisotropy of CMB distribution with a resolution of 13 arcminute - 45 times more sensitive and with 33 times the angular resolution of COBE. These precise data were expected to help understand the geometry, content, and evolution of the Universe, providing finer tests of the Big Bang model. WMAP's measurements played the key role in establishing the current Standard Model of cosmology and have led to the most precise value, till then, of the age of the Universe at $13.75 \pm$ 0.11 billion years; the full timeline of the Universe according to the standard model is given in Figure 5. 


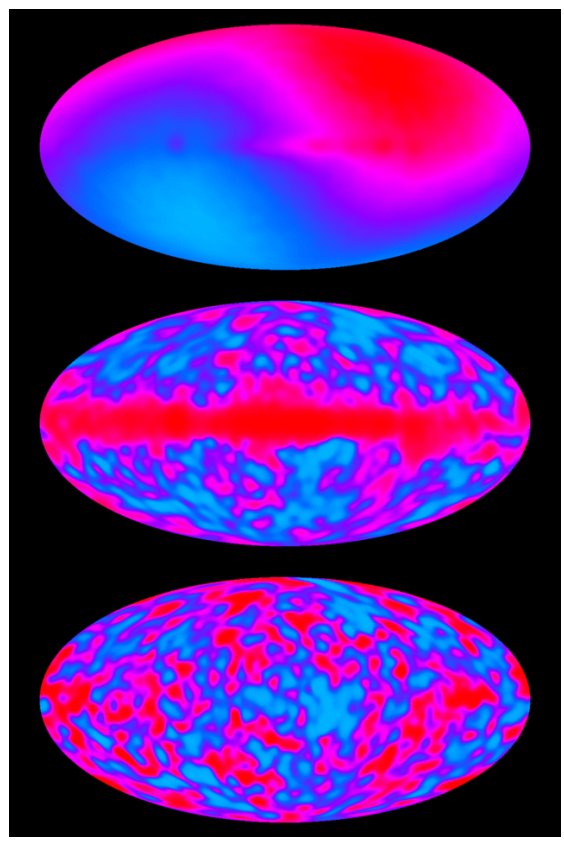

Uncorrected map

Temperature of the Cosmic Microwave Background (CMB) radiation spectrum as determined with the COBE satellite during the first two years of the Differential Microwave Radiometer (DMR) observation. The plane of the Milky Way Galaxy is horizontal across the middle of each picture: (top) uncorrected; (middle) corrected for the dipole term due to our peculiar velocity; (bottom) further corrected to remove the contribution of our galaxy. Note: This map is based on data collected over the two first years of the four-year COBE mission. Therefore, it has been superseded by the four-year map (shown below).

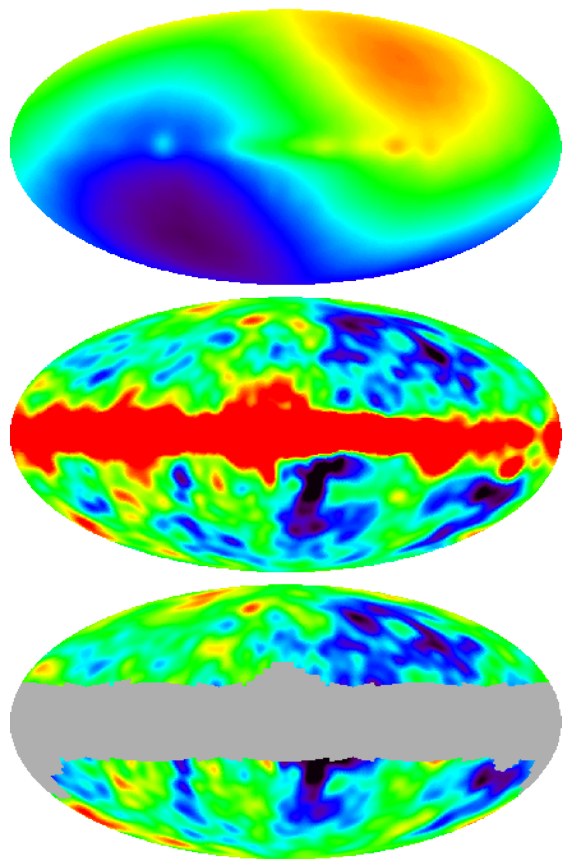

Four-year map

Temperature of the Cosmic Microwave Background (CMB) radiation spectrum as determined with the COBE satellite during the full four years of the Differential Microwave Radiometer (DMR) observation. The plane of the Milky Way Galaxy is horizontal across the middle of each picture. This map shows the $53 \mathrm{GHz}$ channel: (top) prior to dipole subtraction; (middle) after dipole subtraction (due to the solar system movement); (bottom) after subtraction of a model of the Galactic emission.

Figure 4. Source: The COBE datasets were developed by the NASA Goddard space flight center under the guidance of the COBE science working group. http://en.wikipedia.org/wiki/Cosmic Background Explorer 


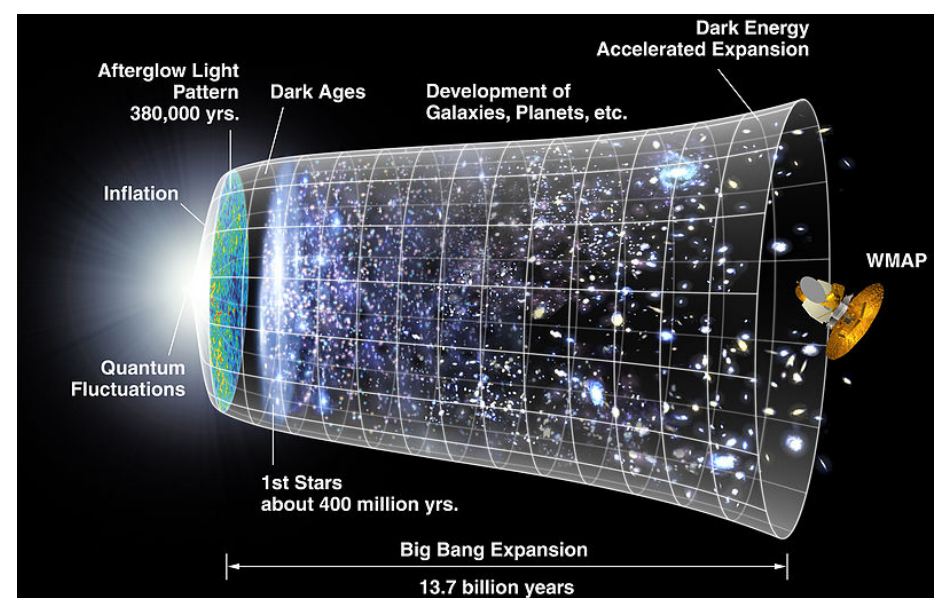

Figure 5. The timeline of the Universe, from inflation to the WMAP. http://en.wikipedia.org/wiki/Wilkinson_Microwave_Anisotropy_Probe

\section{Dark Energy and Dark Matter}

This insight into the nature of the Universe has led to two remarkable new concepts. The first is to do with the expansion of the Universe referred to above. It has all along been expected that the speed of this expansion would slow down as we move farther and farther out in the Universe, since the galaxies would gravitationally pull each other. It was, therefore, to every body's astonishment that, to the contrary, this expansion was found to be accelerating rather than slowing down as we move outwards. This amazing recent discovery based on the observations of the distant Type 1a Supernovae - a late-in-life, dying state of a star —in 1998 [21] [22] caused a great stir among astronomers and physicists alike, leading to the award of the 2011 Nobel Prize in Physics. This novel phenomenon led to the idea of some mysterious "dark energy", which pulls the space apart [23]. The question of the precise nature of this dark energy is one of the hotly debated subjects in astrophysics and of ongoing research in both observational cosmology and theoretical physics.

Another subject of immense current research is an equally, if not more, mysterious object called the "dark matter". This is matter proposed to provide the gravitational glue binding the galaxies together in the cosmos. The original idea for this type of matter arose from the fact that galaxies in some clusters are found to move too fast to be allowed to hold together among themselves; even some stars within some individual galaxies move way too fast for gravity to hold them in these galaxies. Some mysterious invisible "dark matter" was, therefore, proposed to provide the missing gravitational pull in these systems, as early as 1933 [24]. Recent WMAP measurements provided a more direct evidence for this dark matter, however, suggesting that some $83 \%$ of the Universe constituted in "dark matter", while the ordinary matter we see and feel around us in the entire cosmos makes only about $4.56 \%$ of the mass of the Universe [25]-[28].

\subsection{Observation of Dark Matter}

The European Space Agency's (ESA) Planck mission, launched to study the cosmic microwave background (CMB), released the first results after the initial 15 months in March 2013 [29]. These observations revealed new structure in temperature fluctuations of the CMB on a far finer scale than known hitherto (Figure 6). Planck has scanned the entire sky in microwave and submillimeter range of the electromagnetic spectrum, materializing high resolution pictures of the temperature fluctuations since the Universe was very young. The slightly slower expansion rate of the Universe sensed by Planck has extended the age of the Universe slightly, in comparison with the previous estimates-to $13.81 \pm 0.05$ billion years from 13.77 billion years proposed by the WMAP mission and allied observations.

With the initial, opaque plasma state of the Universe after the Big Bang, the excessive energy present did not allow formation of stable atoms until a cooling period of 380,000 years, when an event known as recombination set in. The transparent universe followed with a huge outflow of photons, into the modern era. The initial ultraviolet photons resulting from recombination, however, converted to the microwave spectral region as the universe cooled further down to its current level. The power spectrum of the fluctuations over the sky, revealed by 


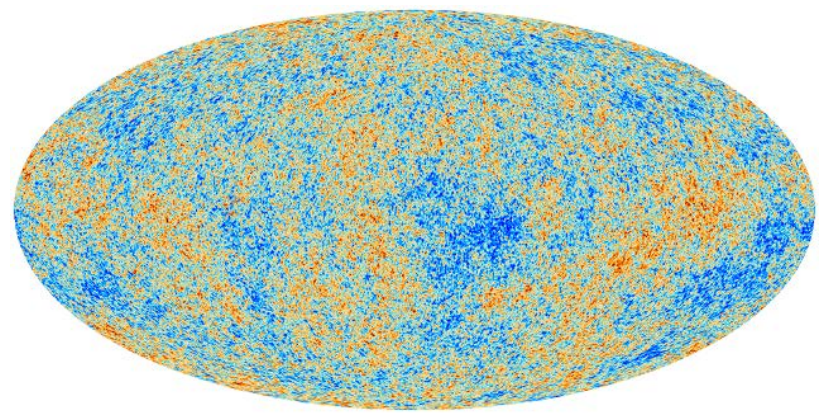

Figure 6. The cosmic microwave background-temperature fluctuations left over from 380,000 thousand years after the Big Bang. This new map is based on data from the Planck mission. ESA/Planck Collaboration/D. Ducros

Planck mission, Figure 7, yields crucial information on the structure and composition of the Universe. The most prominent fluctuations are due to the total energy content of the Universe, while the smaller ones are associated with the distribution of matter alone, both ordinary and dark. The relative magnitudes of the ordinary and dark matter and the conglomeration of the latter are revealed by the smaller fluctuations. Comparison of the Planck data with theoretical models led to finer, but significant adjustments in the hitherto known estimates of energy/matter composition of the Cosmos (Figure 8): dark energy lowered to $68.3 \%$ from $72.8 \%$; dark matter up from $22.7 \%$ to $26.8 \%$; ordinary matter also up from $4.5 \%$ to $4.9 \%$.

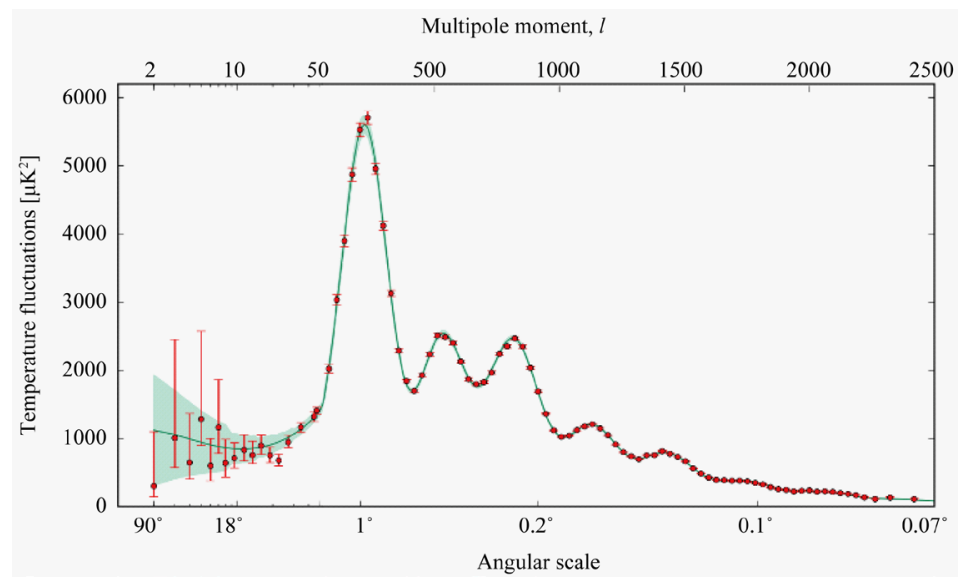

Figure 7. The power spectrum measured by Planck, showing the fluctuations in temperature at a range of size scales on the sky. The anomaly previously seen by WMAP lies at the left edge. The three major peaks show the relative contributions of dark energy, ordinary matter, and dark matter. ESA/Planck Collaboration/D. Ducros

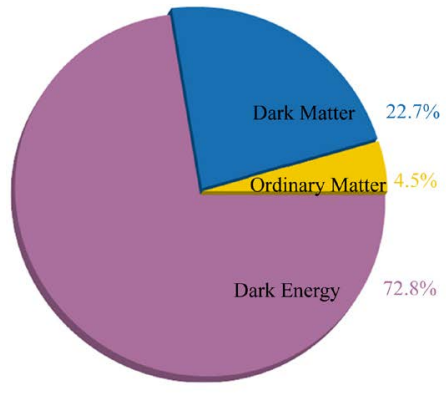

Before Planck

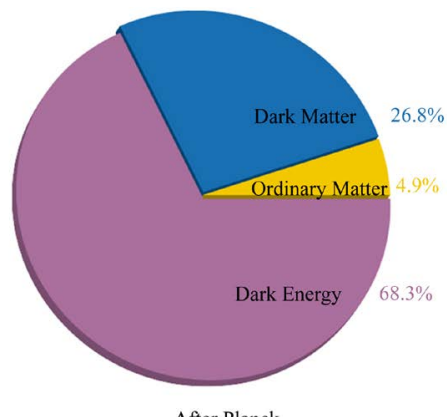

After Planck

Figure 8. The composition of the cosmos, before and after Planck data release. ESA/Planck Collaboration/D. Ducros 


\subsection{Polarization Studies of CMB}

The ultimate evidence for or against dark matter is expected to emerge from the polarization studies of the CMB radiation over the Universe and its anisotropy signature. A great excitement was recently generated by positive news on that count, coming from a telescope station located at the South Pole of the earth. On March 17, 2014 came the announcement of the observation of the so called primordial B-modes by BICEP2 detector of the telescope. These are swirling polarization patterns, Figure 9, in the tiny (1 part in 100,000) fluctuations in CMB temperature across the Universe. These miniscule variations in temperature are interpreted to be due to variations in the density of the primordial gas at the time of emission of the black body radiation, thus reflecting the evolutionary process of the Universe. These fluctuations are thought to be magnified by the gravitational pull of matter, leading to formation of galaxies and clusters of galaxies, now observed in the cosmos. The development of ultrasensitive detectors about a decade back made it possible to provide precise measurements of the polarization, calling in a new era in experimental cosmology and in studies of structure and evolution of the Universe. In particular, the swirling B modes would provide information on the inflationary development - proposed as early as 1980 - of the very early Universe, during the first $10^{-36}$ to $10^{-32}$ seconds of the Big Bang, during which the Cosmos grew by a factor of $10^{26}$. The gravitational waves during this period are supposed to generate this polarization pattern. Dust and magnetic fields in our galaxy were expected to easily mask the tiny polarization signal being sought after. Scales of $1^{\circ}$ used for the initial measurement of B modes were concluded to be large enough to avoid interference due to signals from intervening galaxies. The sceptics found these signals to be far stronger than those predicted or even beyond the limits set by Planck mission on the power of the gravitational waves to generate the CMB temperature fluctuations.

\section{COSMIC CURL}

The BICEP2 instrument observed a faint but distinctive twisting pattern, or spin, known as a curl or B-mode, in the polarization of the cosmic microwave background. This is the first evidence for gravitational waves generated by rapid inflation of the Universe some 13.8 billion years ago.

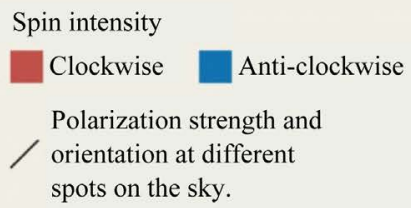

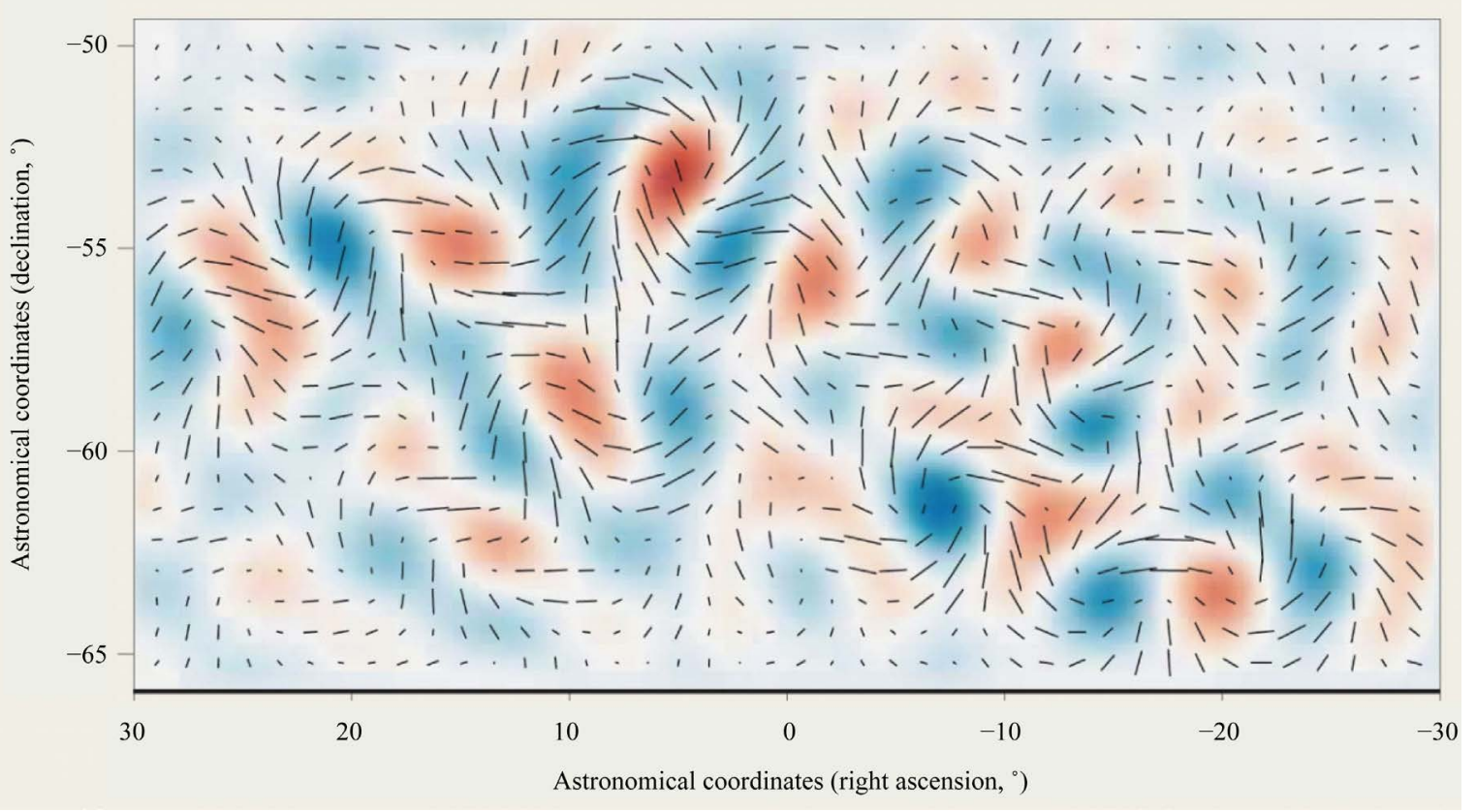

Figure 9. B-mode polarization in the Cosmic Microwave Background (source: BICEP2 Collaboration). http://www.nature.com/news/telescope-captures-view-of-gravitational-waves-1.14876

However, it did not take too long to realize that the twisting CMB polarization pattern could easily be caused by the cosmic dust in the Milky Way galaxy [30]. It has been concluded that the BICEP2 team underestimated 
the contribution of this dust to the swirl polarization pattern, which could almost entirely be accounted for by the latest dust pattern data revealed by Planck mission.

This reversal of the evidence for the detection of primordial gravitational waves applies brakes to the progress in the quest for experimental proof of Big Bang inflationary model, to the idea of multiverse and, of course, to a definitive proof of the existence of dark matter. However, this may signal a temporary hiatus, since, at least, eight experiments, including BICEP3, the Keck Array and Planck, are already focused on this problem. Mean-while, a new perception has emerged regarding the inflationary model, which makes this theoretical model valid irrespective of the experimental evidence for the primordial gravitational waves - the paradigm of inflation would, in that perspective, appear to be unfalsifiable and, therefore, scientifically meaningless [31].

\section{Concluding Remarks}

The brief review above shows clearly how far we have travelled in unraveling the secrets of the Cosmos and the Universe in which we live, over less than a century. It is equally manifest that this rapid progress has been made possible by the enormous advancements in the experimental instrumentation and techniques available for observing the Cosmos. The huge progress in telescopy and the remarkable developments in space technology have made a tremendous impact on the achievable knowledge with high precision and accuracy. While the astronomical knowledge of the cosmos has witnessed impressive progress, the analysis of these observations has opened new frontiers in our understanding of the fundamental physics and nature and evolution of the Universe.

It is clear from the above brief description of the current state of our knowledge of the Universe that the age old maxim, the more we know, less we conclude we actually know, appears to be perfectly valid. However, such is perhaps the nature of all human knowledge. But how exciting the ever expanding frontiers of this field of fundamental physics are can be gauged from this brief narrative. It is also clear that our quest for the knowledge of the Universe will be a continuing chapter in the story of human search and discovery for the years to come and may yet raise more questions than it can answer. Thanks to the remarkable developments in technology, and hence the tools of observational astronomy, many new frontiers of the unknown may yet be opened for even more extensive exploration of the Cosmos than the territory conquered hitherto.

\section{References}

[1] Jansky, K.G. (1933) Radio Waves from Outside the Solar System. Nature, 132, 66. http://dx.doi.org/10.1038/132066a0

[2] Hey, J.S. (1983) The Radio Universe. Vol. 1, Oxford Pergamon Press, Oxford, 253.

[3] Bolton, J.G., Stanley, G.J. and Slee, O.B. (1949) Positions of Three Discrete Sources of Galactic Radio-Frequency Radiation. Nature, 164, 101-102. http://dx.doi.org/10.1038/164101b0

[4] Barath, F.T., Barrett, A.H., Copeland, J., Jones, D.E. and Lilley, A.E. (1963) Microwave Radiometers. Science, 139, 908-909. http://dx.doi.org/10.1126/science.139.3558.908

[5] Burke, B.F. and Franklin, K.L. (1955) Observations of a Variable Radio Source Associated with the Planet Jupiter. Journal of Geophysical Research, 60, 213-217. http://dx.doi.org/10.1029/JZ060i002p00213

[6] Muller, C.A. and Oort, J.H. (1951) Observation of a Line in the Galactic Radio Spectrum: The Interstellar Hydrogen Line at $1420 \mathrm{Mc} . / \mathrm{sec}$. , and an Estimate of Galactic Rotation. Nature, 168, 357-358. http://dx.doi.org/10.1038/168357a0

[7] Ewen, H.I. and Purcell, E.M. (1951) Observation of a Line in the Galactic Radio Spectrum. Nature, 168, 356-358.

[8] Chen, W., Gehrels, N., Diehl, R. and Hartmann, D. (1996) On the Spiral Arm Interpretation of COMP ${ }^{\mathrm{TeL}} \wedge 26^{\wedge} \mathrm{Al}^{\mathrm{Map}}$ Features. Astronomy and Astrophysics Supplement Series, 120, 315.

[9] Russeil, D. (2003) Star-Forming Complexes and the Spiral Structure of Our Galaxy. Astron. Astrophys.-Berl.-, 397, 133-146.

[10] Levine, E.S., Blitz, L. and Heiles, C. (2006) The Spiral Structure of the Outer Milky Way in Hydrogen. Science, 312, 1773-1777.

[11] Churchwell, E., Babler, B.L., Meade, M.R., Whitney, B.A., Benjamin, R., Indebetouw, R., Cyganowski, C., Robitaille, T.P., Povich, M., Watson, C. and Bracker, S. (2009) The Spitzer/GLIMPSE Surveys: A New View of the Milky Way. Publications of the Astronomical Society of the Pacific, 121, 213-230. http://dx.doi.org/10.1086/597811

[12] Jennison, R.C. and Das Gupta, M.K. (1953) Fine Structure of the Extra-Terrestrial Radio Source Cygnus 1. Nature, 172, 996-997. http://dx.doi.org/10.1038/172996a0

[13] Baade, W. and Zwicky, F. (1934) Cosmic Rays from Super-Novae. Proceedings of the National Academy of Sciences 
of the United States of America, 20, 259-263. http://dx.doi.org/10.1073/pnas.20.5.259

[14] Schmidt, M. (1963) 3C 273: A Star-Like Object with Large Red-Shift. Nature, 197, 1040. http://dx.doi.org/10.1038/1971040a0

[15] Matthews, T.A. and Sandage, A.R. (1963) Optical Identification of 3c 48, 3c 196, and 3c 286 with Stellar Objects. Astrophysical Journal, 138, 30.

[16] Greenstein, J.L. and Schmidt, M. (1964) The Quasi-Stellar Radio Sources 3c 48 and 3c 273. Astrophysical Journal, 140, 1. http://dx.doi.org/10.1086/147889

[17] Hewish, A., Bell, S.J., Pilkington, J.D.H., Scott, P.F. and Collins, R.A. (1968) Observation of a Rapidly Pulsating Radio Source. Nature, 217, 709-713. http://dx.doi.org/10.1038/217709a0

[18] Penzias, A.A. and Wilson, R.W. (1965) A Measurement of Excess Antenna Temperature at 4080 Mc/s. Astrophysical Journal, 142, 419-421. http://dx.doi.org/10.1086/148307

[19] Boggess, N.W., Mather, J.C., Weiss, R., Bennett, C.L., Cheng, E.S., Dwek, E., Gulkis, S., Hauser, M.G., Janssen, M.A., Kelsall, T., et al. (1992) The COBE Mission-Its Design and Performance Two Years after Launch. Astrophysical Journal, 397, 420-429. http://dx.doi.org/10.1086/171797

[20] Cosmic Background Explorer. Wikipedia, the free encyclopedia, 21 July 2014.

[21] Riess, A.G., Filippenko, A.V., Challis, P., Clocchiatti, A., Diercks, A., Garnavich, P.M., Gilliland, R.L., Hogan, C.J., Jha, S., Kirshner, R.P., et al. (1998) Observational Evidence from Supernovae for an Accelerating Universe and a Cosmological Constant. Astronomical Journal, 116, 1009. http://dx.doi.org/10.1086/300499

[22] Perlmutter, S., Aldering, G., Goldhaber, G., Knop, R.A., Nugent, P., Castro, P.G., Deustua, S., Fabbro, S., Goobar, A., Groom, D.E., et al. (1999) Measurements of $\Omega$ and $\Lambda$ from 42 High-Redshift Supernovae. Astrophysical Journal, 517, 565. http://dx.doi.org/10.1086/307221

[23] Peebles, P.J.E. and Ratra, B. (2003) The Cosmological Constant and Dark Energy. Reviews of Modern Physics, 75, 559. http://dx.doi.org/10.1103/RevModPhys.75.559

[24] Zwicky, F. (2009) Republication of: The Redshift of Extragalactic Nebulae. General Relativity and Gravitation, 41, 207-224. http://dx.doi.org/10.1007/s10714-008-0707-4

[25] Ade, P.A.R., Aghanim, N., Armitage-Caplan, C., Arnaud, M., Ashdown, M., Atrio-Barandela, F., Aumont, J., Baccigalupi, C., Banday, A.J., Barreiro, R.B., et al. (2013) Planck 2013 Results. I. Overview of Products and Scientific Results. arXiv:1303.5062.

[26] Francis, M. (2013) First Planck Results: The Universe Is Still Weird and Interesting. Ars Technica, 21 March 2013. http://arstechnica.com/science/2013/03/first-planck-results-the-universe-is-still-weird-and-interesting/

[27] First Observational Evidence of Dark Matter. http://www.darkmatterphysics.com/Galactic-rotation-curves-of-spiral-galaxies.htm

[28] Dark Matter. Wikipedia, the free encyclopedia, 22 July 2014.

[29] Cowen, R. (2014) Telescope Captures View of Gravitational Waves. Nature, 507, 281-283. http://dx.doi.org/10.1038/507281a

[30] Cowan, R. (2014) Big Bang Finding Challenged. Nature, 510, 20. http://dx.doi.org/10.1038/510020a

[31] Steinhardt, P. (2014) Big Bang Blunder Bursts the Multiverse Bubble. Nature, 510, 9. http://dx.doi.org/10.1038/510009a 